\title{
A tale of three cities
}

\section{OmegaCAM discovers multiple sequences in the color-magnitude diagram of the Orion Nebula Cluster}

\author{
G. Beccari ${ }^{1}$, M. G. Petr-Gotzens ${ }^{1}$, H. M. J. Boffin ${ }^{1}$, M. Romaniello ${ }^{1,12}$, D. Fedele ${ }^{2}$, G. Carraro ${ }^{3}$, G. De Marchi ${ }^{4}$, \\ W.-J. de Wit ${ }^{5}$, J. E. Drew ${ }^{6}$, V. M. Kalari ${ }^{7}$, C. F. Manara ${ }^{4}$, E. L. Martin ${ }^{8}$, S. Mieske ${ }^{5}$, N. Panagia ${ }^{9}$, L. Testi ${ }^{1}$, J. S. Vink ${ }^{10}$, \\ J. R. Walsh ${ }^{1}$, and N. J. Wright ${ }^{6,11}$
}

${ }^{1}$ European Southern Observatory, Karl-Schwarzschild-Strasse 2, 85748 Garching bei München, Germany e-mail: gbeccari@eso.org

2 INAF-Osservatorio Astrofisico di Arcetri, L.go E. Fermi 5, 50125 Firenze, Italy

3 Dipartimento di Fisica e Astronomia Galileo Galilei, Vicolo Osservatorio 3, 35122 Padova, Italy

${ }^{4}$ Science Support Office, Directorate of Science, European Space Research and Technology Centre (ESA/ESTEC), Keplerlaan 1, 2201 AZ Noordwijk, The Netherlands

5 European Southern Observatory, Alonso de Córdova 3107, 19001 Casilla Santiago, Chile

6 School of Physics, Astronomy and Mathematics, University of Hertfordshire, College Lane Campus, Hatfield, AL10 9AB, UK

7 Departamento de Astronomía, Universidad de Chile, Casilla 36-D Correo Central, Santiago, Chile

8 CSIC-INTA Centro de Astrobiologia Carretera Ajalvir km 4, 28550 Madrid, Spain

9 Space Telescope Science Institute, 3700 San Martin Drive, Baltimore, MD 21218, USA

10 Armagh Observatory, College Hill, Armagh BT61 9DG, UK

11 Astrophysics Group, Keele University, Keele ST5 5BG, UK

12 Excellence Cluster Universe, Garching bei München, Germany

Received 13 January 2017 / Accepted 26 May 2017

\section{ABSTRACT}

As part of the accretion discs in $\mathrm{H} \alpha$ with OmegaCAM (ADHOC) survey, we imaged a region of $12 \times 8$ square degrees around the Orion Nebula Cluster in $r, i$ and $\mathrm{H} \alpha$. Thanks to the high-quality photometry obtained, we discovered three well-separated pre-main sequences in the color-magnitude diagram. The populations are all concentrated towards the cluster's center. Although several explanations can be invoked to explain these sequences, we are left with two competitive but intriguing scenarios: a population of unresolved binaries with an exotic mass ratio distribution, or three populations with different ages. Independent high-resolution spectroscopy supports the presence of discrete episodes of star formation, each separated by about a million years. The stars from the two putative youngest populations rotate faster than the older ones, in agreement with the evolution of stellar rotation observed in pre-main sequence stars younger than $4 \mathrm{Myr}$ in several star forming regions. Whatever the final explanation, our results prompt a revised look at the formation mode and early evolution of stars in clusters.

Key words. stars: pre-main sequence - open clusters and associations: individual: Orion

\section{Introduction}

Young stellar clusters are conspicuous components of our Galaxy. They are the best test beds of the stellar initial mass function because they are assumed to be entities of a common origin, that is, born from the same molecular cloud material, and at the same time. Observations have shown, however, that the stars born in one and the same cluster are not as coeval as expected. In fact, stellar age spreads up to several million years have been postulated for several clusters from fitting isochronal ages to the position of cluster-member stars in the Hertzsprung-Russell diagram (HRD; e.g., Cargile \& James 2010; Cignoni et al. 2010; Reggiani et al. 2011; Bell et al. 2013; Balog et al. 2016). However, whether this observation is a consequence of a continuous star formation process lasting over several dynamical time scales, or is rather caused by different accretion histories within an otherwise coeval population of pre-main sequence (PMS) stars, is widely debated (Da Rio et al. 2010; Jeffries et al. 2011).
The Orion Nebula Cluster (ONC) is the nearest (414 pc; Menten et al. 2007) populous young stellar cluster and hence the best laboratory for testing the existence of stellar age spreads. Palla et al. $(2005,2007)$ were the first to point out an apparent large age spread of $\sim 10 \mathrm{Myr}$ based on the evidence of lithium depletion in some stellar cluster members. Da Rio et al. (2010) interprets the luminosity spread of the ONC's PMS as an age spread of 0.3-0.4 dex with a mean age of 3-4 Myr depending on the models.

In this article we present a photometric study of a $12^{\circ} \times 8^{\circ}$ area including the ONC. We report the detection for the first time of multiple sequences in the observed optical color-magnitude diagram of the ONC.

\section{Observations and data reduction}

The images used in this work were collected with the widefield optical camera OmegaCAM on the 2.6-m VLT Survey Telescope (VST) at Cerro Paranal in Chile. OmegaCAM consists of 
a mosaic of 32 CCDs and samples a $1 \mathrm{deg}^{2}$ field of view (FoV) with a pixel sampling of $0.21 \mathrm{arcsec}_{\mathrm{pixel}}{ }^{-1}$. Orion was sampled through the $r, i$ broad-band filters and the $\mathrm{H} \alpha$ narrow band as part of the Accretion Discs in $\mathrm{H} \alpha$ with OmegaCAM (ADHOC) survey (PI: Beccari). Each target region is sampled in groups of three overlapping fields, and in each group the fields are contiguous with a footprint close to $3 \times 1 \mathrm{deg}^{2}$. For each position in sky we acquire two exposures of $25 \mathrm{sec}$ in $r$ and $i$ and three images of $150 \mathrm{sec}$ exposure with the $\mathrm{H} \alpha$ filter. The frames were collected between October and December 2015 and the median image quality is $0.91 \pm 0 . ' 16$.

The pattern to these observations is similar to that of the VPHAS + survey, and the data have passed through the same pipeline (see Drew et al. 2014, for more details). The entire dataset was fully processed, from the bias, flat-field, and linearity correction to the stellar photometry at the Cambridge Astronomical Survey Unit (CASU). The magnitude for each star is extracted using aperture photometry adopting an algorithm based on IMCORE ${ }^{1}$ (Irwin 1985) and the nightly photometric calibrations are also performed. We downloaded the astrometrically and photometrically calibrated single band catalogs from the VST archive at CASU ${ }^{2}$. Stars lying in the overlap region between adjacent fields were used to adjust residual photometric offsets. The photometric calibration of the final band-merged catalog covering the entire area was checked against a catalog of stars from the AAVSO Photometric All Sky Survey, used as a secondary standard catalog. The final catalog allows us to homogeneously sample the stellar populations in a region of $12^{\circ} \times 8^{\circ}$ size in Orion down to $r \sim 20$.

\section{Three distinct pre-main sequences in the ONC}

We show in Fig. 1a the $(r-i)$ versus $r$ color-magnitude diagram (CMD) of the stars located inside a radius of 1.5 from the center of the ONC. The population of PMS objects is well detected and occupies the reddest side of the CMD above the population of back/foreground stars in the magnitude range $14<r<20$ and $(r-i)>1$, which roughly corresponds to PMS stars of masses between 0.2 and $1 M_{\odot}$ (see also Da Rio et al. 2009, 2016). A remarkable feature is well visible on the CMD shown in Fig. 1a, that is, the presence of at least two distinct and near-parallel sequences of PMS stars. Following De Marchi et al. (2016), in Fig. 1b we have applied to the CMD the "unsharp-masking" technique of Spiegler \& Juris (1931) in order to enhance the high-frequency features in the diagram. The result shown in Fig. 1b further supports the detection of at least two distinct PMSs in the CMD diagram. We stress here that by inspecting the entire surveyed area we found that this feature is clearly detectable only in the ONC region.

We used a simplified version of the method described in Milone et al. (2009) to further investigate the existence of two or more distinct sequences in the CMD. In Fig. 2a we show a portion of the CMD zoomed on the population of the PMS. In order to increase the contrast of the ONC population against the back/foreground stars, we show only stars inside a radius of 0.5 from the center of the cluster. The black line shows the mean ridge line of the blue PMS. We then calculate the distance in $r-i$ color of each star in a magnitude range $15.5<r<16.5$ from the mean ridge line arbitrarily chosen as a reference line. We adopted this magnitude range to provide an adequate statistical balance between the number of stars in the PMS population and

\footnotetext{
1 Software publicly available from http://casu.ast. cam.ac.uk

2 http://casu.ast.cam.ac.uk/vstsp/
}

the contamination from field stars. The distance of each star as a function of the $r$ magnitude is shown on panel (b) of the same figure. Panel (c), the histogram of the distances in $(r-i)$ color, clearly shows the presence of three distinct populations of PMS stars well separated in color on the CMD. Indeed the Hartigans' dip test confirms that the distribution of the color distances is incompatible with a uni-modal's one. The number fraction of stars belonging to the two populations with the reddest colors (green and red in the figure) compared to the reference one (blue) is 0.5 and 0.15 , respectively. These fractions hold (within the statistical uncertainties) even after accounting for contamination, which we estimated to be between 15 and $40 \%$ based on the CMD of a control field located a few degrees west of the ONC, and spectroscopic membership information from APOGEE spectra (see Sect. 6).

Next, we investigate the spatial distribution of stars belonging to the different sequences. In Fig. 3 we show the surface densities of the three populations. These density plots are calculated using all the stars belonging to the three PMS populations selected in the color and magnitude ranges shown in the CMD of Fig. 2. We calculate the densities using the GATHER method from Gladwin et al. (1999). In the maps of Fig. 3 we show the position of the stars with respect to the ONC nominal center (solid circles) together with the density contours, which were scaled to the maximum value of each population. It emerges that the density distributions of the three populations all peak around a common center. The blue population seems to be slightly more sparsely distributed with respect to the spatial distribution of the stars belonging to the green and red populations.

We used the Minimal Spanning Tree (MST; Allison et al. 2009) to assess if any difference in the spatial distribution of the populations is present and at what level of significance. The MST is the unique set of straight lines ("edges") connecting a given sample of points ("vertices"; in this case the star coordinates) without closed loops, such that the sum of the edge lengths is the minimum possible. Hence, the length of the MST is a measure of the compactness of a given sample of vertices (Cartwright \& Whitworth 2004). The MST is a powerful algorithm to study population distributions since it can be used without assuming that the studied populations are distributed around the same center of gravity, which is mandatory when using the Kolmogorow-Smirnov (KS) test. The weakness of this method is that it must be assumed that the photometric completeness of the compared populations is the same. We have selected the populations in a common range of magnitudes that are affected by the same level of completeness. The fact that the three populations are affected by the same contamination from field stars, makes it impossible to use the MST when the number of genuine members is low, which is the case for the red population. For this reason we limit the use of the MST to the blue and green populations.

We first estimate the MST of all the stars in the green population. Then we randomly extract 1000 sets of stars belonging to the blue population equal in number to the number of stars in the green population. We compute the MST of the blue populations as the mean and the standard deviation of the distribution of 1000 MSTs. Hence, we calculate ${ }^{3}$

$\Lambda M S T_{\text {blue }}=\frac{M S T_{\text {blue }}}{M S T_{\text {green }}}$.

3 We highlight here that $\Lambda M S T=1$ means that the compared populations are equally distributed in space while a value greater than one indicates that the population at the numerator is more spatially extended than the one at the denominator. 

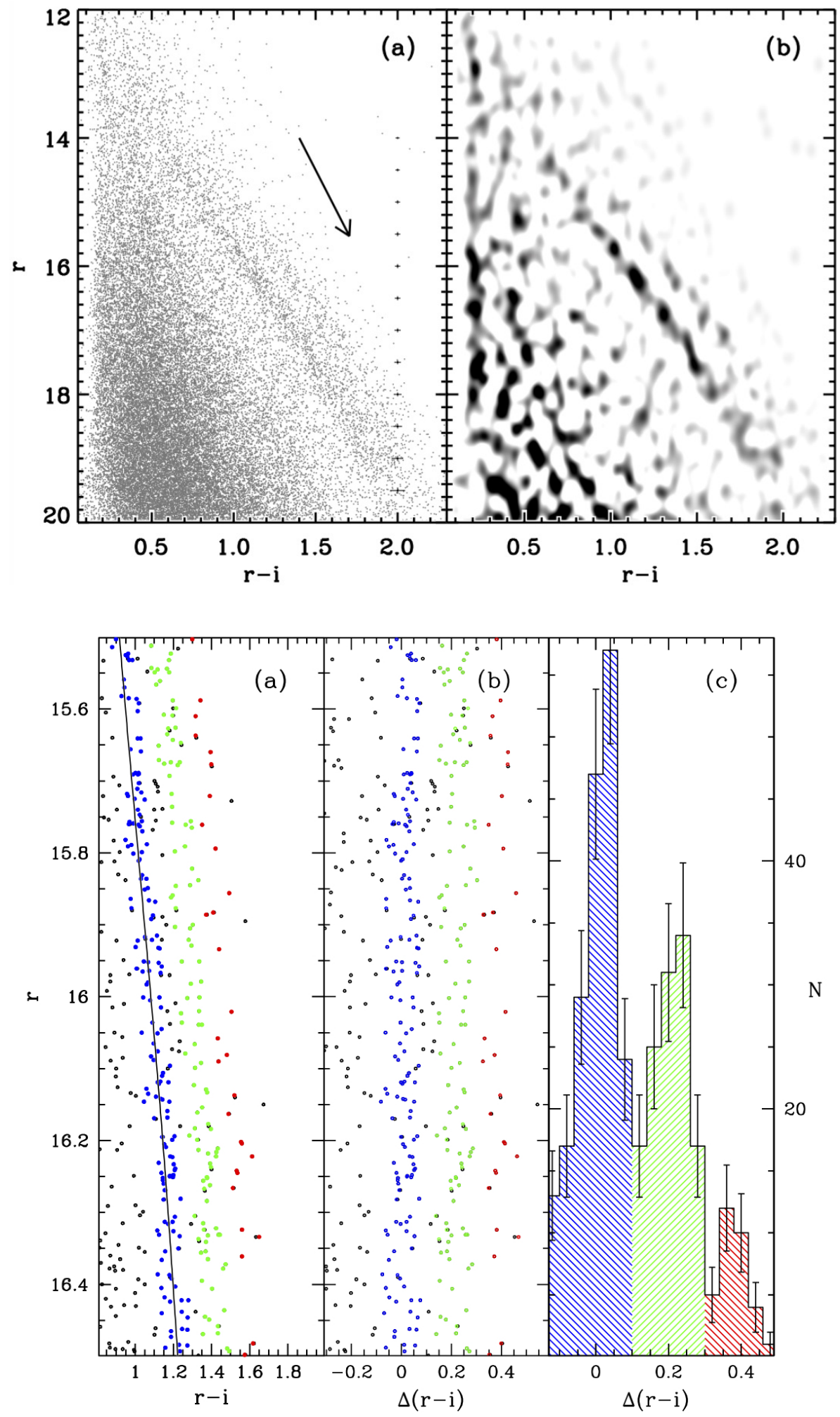

Fig. 1. a) The $(r-i), r$ color-magnitude diagram of a region of 1.5 radius centered on the ONC. The arrow represents a reddening vector for $A_{\mathrm{v}}=1.8$ (typical for Orion; Schlafly et al. 2014) and extinction curve with $R_{\mathrm{v}}=3.1$ (Cardelli et al. 1989). The photometric errors (magnitudes and colors) are indicated by black crosses; b) the same CMD shown in a) after unsharp masking.
Fig. 2. a) Portion of the CMD zoomed on the PMS. The black line shows the mean ridge line of the blue population; b) rectification of the CMD shown in panel a); c) histogram of the distance in $(r-i)$ color of the PMS stars from the mean ridge line of the bluest population.
We find that $\Lambda M S T_{\text {blue }}=1.16 \pm 0.03$, which indicates that the blue sequence is slightly more sparsely distributed with respect to the green one with a $5 \sigma$ significance.

\section{The unresolved binaries/multiples scenario}

The appearance of multiple parallel sequences in the CMD as shown in Fig. 1 could be caused by (1) a single coeval population consisting of single stars and unresolved binaries and higher order multiples; or populations (2) at different distances; (3) with different extinction $A_{\mathrm{v}}$; or (4) with different ages. In this and the following sections we discuss these four hypotheses.

Any unresolved binary system would appear in a CMD as a single star with a flux equal to the sum of the fluxes of the two components. This effect produces a systematic over-luminosity of these objects and a shift in color which depends on the 


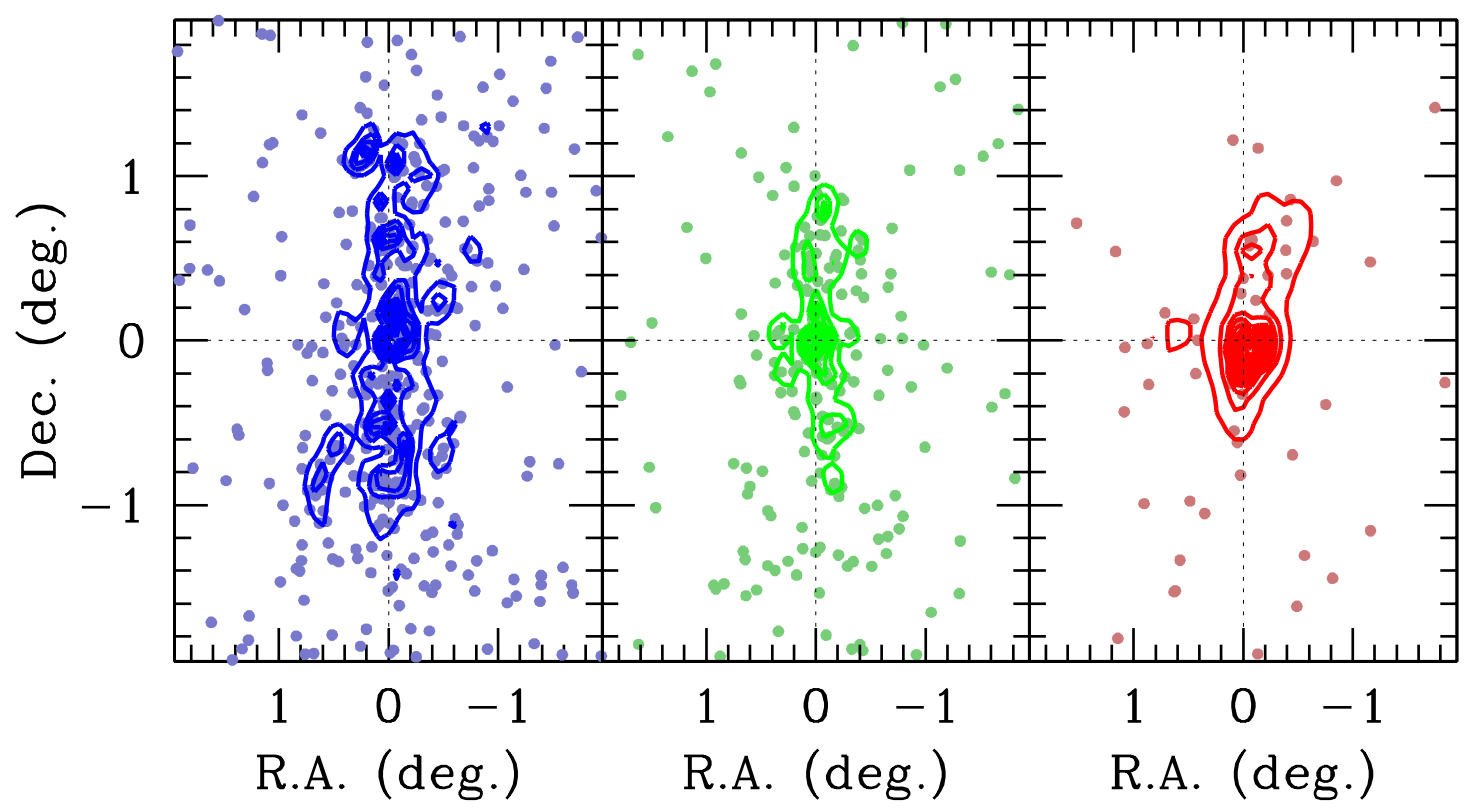

Fig. 3. Surface density of the three populations, old (in blue), young (in green), and very young (in red) together with the position of the stars. The plots have been centered on the ONC nominal center. All contours are normalized to the maximum value of the population itself. The location of the stars belonging to each population (solid dots) are also shown.

magnitudes (and hence mass) of the two components in each passband. When the mass ratio of two stars in the binary system is $q=1$ (equal mass binary), the unresolved binary system appears 0.752 mag brighter than the individual component's brightness. The peak of the distribution of the green population is $\sim 0.75$ mag brighter than the blue population (Fig. 1a) and this distribution may therefore well reflect the presence of a population of unresolved ONC binaries. Under the assumption that the green and red sequences represent the binaries and higher-order multiples of the ONC members, we derive a multiplicity fraction of $39 \%$. This number would support the unresolved binary hypothesis given that this fraction overall agrees with that seen for low-mass stars in other young clusters (Luhman et al. 2005; Reipurth et al. 2014) and with the multiplicity fraction among field M-dwarfs (Duchêne \& Kraus 2013).

Besides the total fraction, the companion mass ratio distribution plays an important role in shaping the CMD appearance. Determinations of the mass ratio distribution for ONC binaries are rare. Those studies that have derived mass ratios for close visual systems and spectroscopic systems find no indication for an equal mass preference (Daemgen et al. 2012; Correia et al. 2013; Kounkel et al. 2016), that is, for those systems that lead to the largest displacement in luminosity in the CMD. At most, the observed mass ratio distribution slightly increases from low $q$ to higher $q$ (Correia et al. 2013), although this result is most likely affected by incompleteness and selection effects at low $q$. Ward-Duong et al. (2015) performed a companion survey of 245 late-K to mid-M (K7-M6) dwarfs within $15 \mathrm{pc}$ and found that the mass ratio distribution across the $q=0.2-1.0$ range is flat. This seems to be a general result. Numerical simulations of primordial binaries (e.g. Bate 2009) produce an $f(q)$ that is rather flat too. Moreover $f(q)$ seems to be rather insensitive to dynamical disruptions and interaction processes within the cluster (Parker \& Reggiani 2013).

The marked CMD morphology, and in particular the presence of the two gaps in the distribution of colors shown in
Fig.2c, allows us to investigate which combination of total binary frequency and companion mass ratio distribution could explain the observed CMD, and which can be excluded. For this, we performed Monte Carlo simulations and tested a range of total binary fractions and mass ratio distributions $f(q)^{4}$. We randomly draw a star from the blue sequence (with its $r$ and $i$ magnitudes) and, using the mass-luminosity relation from Bressan et al. (2012), we determine its mass. We then draw a mass ratio from a given $f(q)$ distribution, and add a companion with a mass $q m_{1}$. We then compute the color and magnitude of the resulting binary and can then see where the thus obtained binary falls in the CMD. Finally, we compare the obtained histograms of colors with that observed in Fig.2c. A range from $40 \%$ to $100 \%$ in binary fraction and different $f(q)$ of the form uniformly flat, linearly increasing, quadratically increasing, or step-like were explored. In Fig. 4 we show a number of representative results.

It is clear that the canonical case of $40 \%$ binary fraction with a uniform mass-ratio distribution, as shown in the upper panels of Fig. 4, does not provide a satisfactory agreement with the observations. Increasing the fraction of binaries even worsens the comparison with observations, as does a lower total binary fraction. We conclude that a uniformly flat mass ratio distribution is not able to reproduce the observations, in particular it is not capable of reproducing the obvious, significant gaps in the CMD. When assuming other mass-ratio distributions we can achieve reasonable fits (e.g., second to fourth-row panels in Fig. 4). Here we considered as reasonable fit any solution with a reduced $\chi^{2}<2$ which indicates that it can be trusted with $99.5 \%$ confidence. The solutions come with some caveats, however. A linearly increasing $f(q)$ (second-row panels of Fig. 4) appears only possible in combination with a total binary fraction of around $60 \%$. However, such high overall binary frequencies among ONC low-mass stars are not observed. For visual

\footnotetext{
4 We note that when doing this, we only use the blue and green populations, and ignore for now the red population.
} 


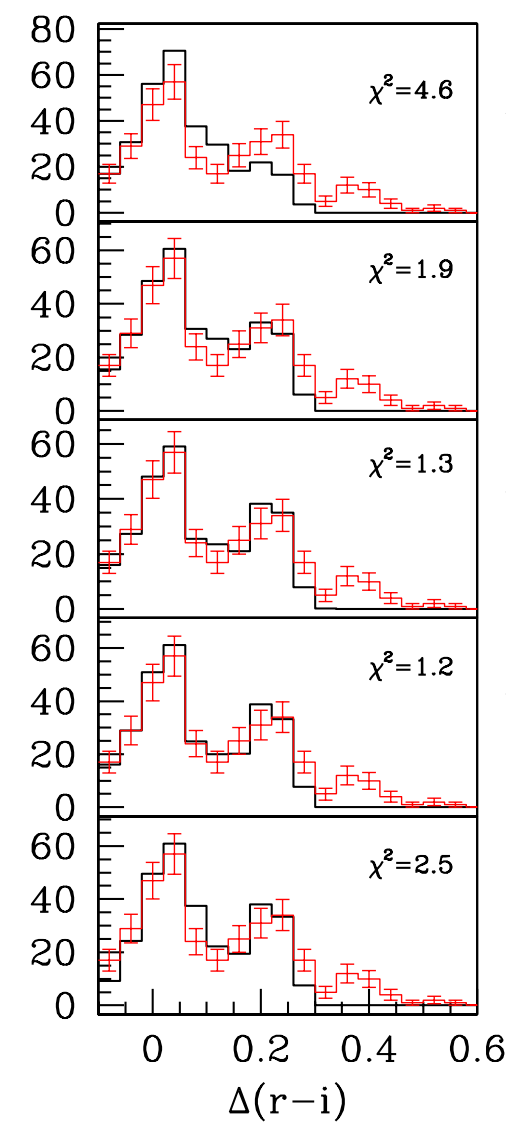

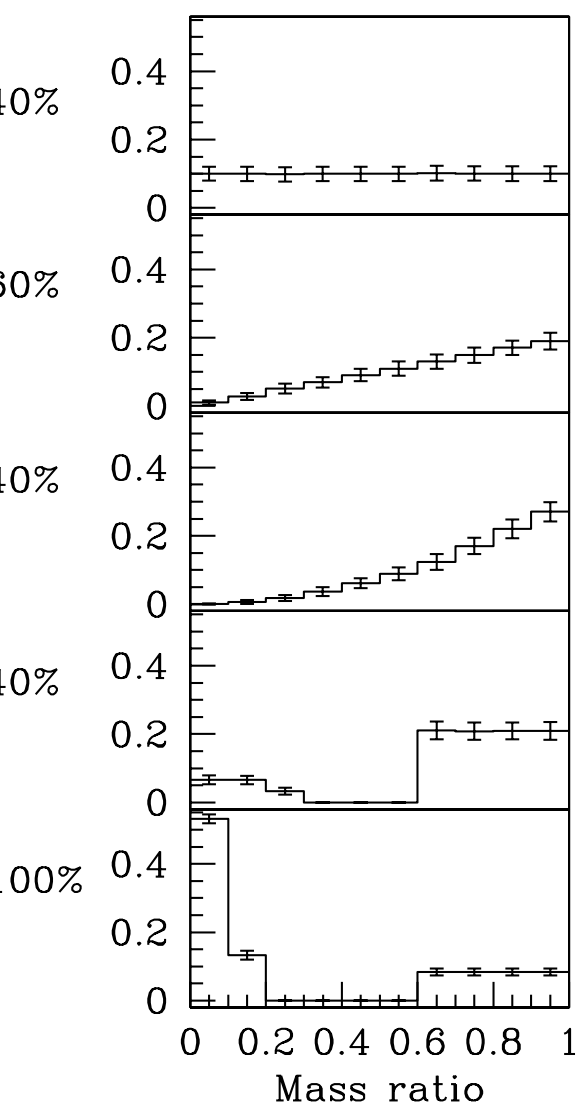

Fig. 4. Left panels: histograms of the distance in the $(r-i)$ color of the PMS stars from the mean ridge line of the bluest population, as obtained with our Monte Carlo simulations (black, heavy lines) and as observed (red). Right panels: corresponding assumed distribution of the mass ratios of all the binaries in our simulated sample. The $\chi^{2}$ of each solution is also indicated in the left panels. Both top panels correspond to the canonical cases of $40 \%$ multiplicity and a uniform mass ratio distribution. binaries with separations between a few tens and a few hundreds of AU (which would indeed appear unresolved in our OmegaCAM observations) various studies have consistently found that the ONC binary fraction is even slightly lower than in the field (Petr et al. 1998; Köhler et al. 2006; Reipurth et al. 2007). Also very close binaries with separations $<10 \mathrm{AU}$ that have been traced by a multi-epoch spectroscopic study (Tobin et al. 2013; Kounkel et al. 2016) do not show any excess in companions, but are again consistent with field star fractions. Hence, the overall binary fraction cannot noticeably exceed $40 \%$ unless these excess binaries are all in the narrow separation range of 10-40 AU which seems very unlikely. On the other hand, if we enforce mass ratio distributions that are strongly skewed towards high mass ratios, such as a quadratically increasing or step-like function where the majority of systems would have $q>0.6$, then a simulated population with a total binary fraction as low as $35-40 \%$ can reproduce the observations (third and fourth row panels of Fig. 4). Actually, also higher total binary fractions, up to $65 \%$ would be feasible according to our simulation. In this case, however, the blue population would contain a large number of unresolved low $q$ systems. Such specific mass ratio distributions are at odds with observations. We are thus led to the conclusion that in order for the binary hypothesis to be valid, one would need to postulate a very distinct mass-ratio distribution.

In the same vein, the reddest sequence we find in the CMD would be even more difficult to explain, as it would require triple systems, where the mass ratio distributions would also need to be quite tailored, that is, the mass ratios of both secondaries and tertiaries would need to be strongly peaked to $q>0.8$ and be highly similar.
Although formally we cannot exclude the presence of a population of binaries with an unusual $f(q)$, the fact that no young binary population in any star forming region has shown indications of such a usual $f(q)$, casts some doubts on binaries as the origin for the observed multiple sequences. On the other hand, if confirmed, this fact would certainly challenge many of the studies published so far on the stellar population in the ONC whenever such stars were considered as single objects (e.g. Hillenbrand et al. 2013; Da Rio et al. 2016).

\section{Differential $\boldsymbol{A}_{\mathrm{v}}$ and distances scenario}

Any effect of differential extinction can be rejected by the fact that the reddening vector as shown on the CMD of Fig. 1a runs parallel to the PMSs.

Moreover, as shown in Fig. 5 we have verified that the stars from the three sequences have approximately the same distribution of visual extinction $A_{\mathrm{v}}$. For this comparison we adopted the $A_{\mathrm{v}}$ provided by the spectroscopic analysis of Da Rio et al. (2016).

An alternative explanation for the presence of the green and red sequences could be a PMS population located more than 100 and $200 \mathrm{pc}$ in the foreground of the blue sequence, respectively. Alves \& Bouy (2012) suggested that the population of PMS around $\iota$ Ori belongs to the association NGC 1980 and represents a population of 4-5 Myr old stars located at $\sim 30 \mathrm{pc}$ in the foreground of Orion A (Bouy et al. 2014). In their detailed spectroscopic analysis Da Rio et al. (2016) rule out this hypothesis, finding that the candidate foreground population is kinematically indistinguishable from the Orion A's one. They conclude that the old population studied by Bouy et al. (2014) witnesses the earliest (i.e., oldest) episode of star formation in 


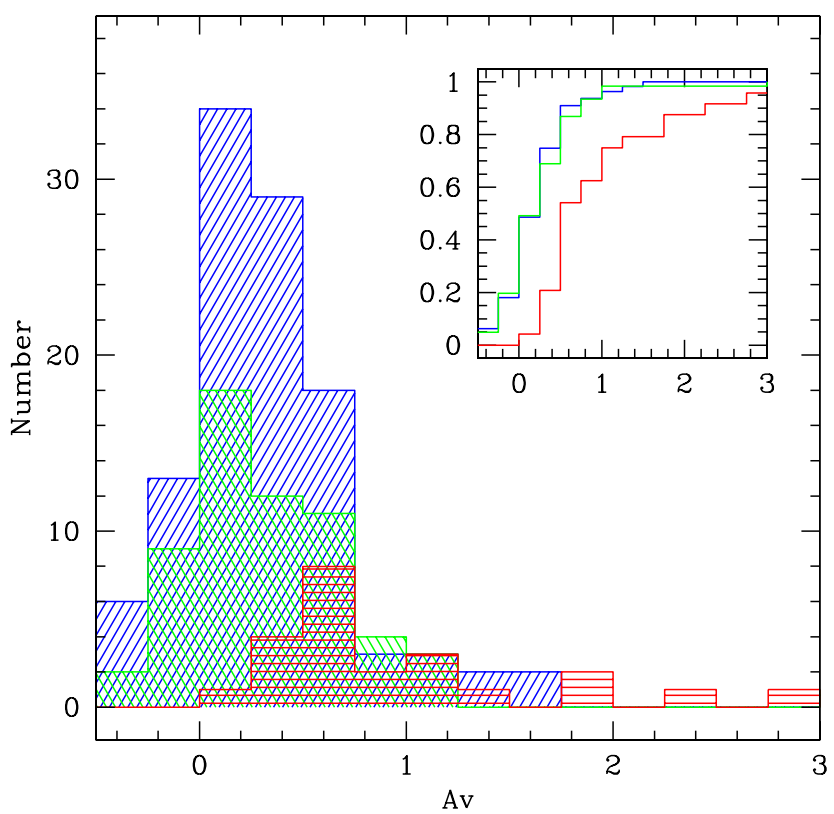

Fig. 5. Distribution of the visual extinction, $A_{\mathrm{v}}$, for the stars from the three populations, old (in blue), young (in green), and very young (in red). The inset shows the normalized cumulative fraction. The Kolmogorov-Smirnov test indicates that the $A_{\mathrm{v}}$ distribution of the old and intermediate-young populations are extracted from the same parental distribution with more than $5 \sigma$ significance. The very young population shows signs of a slightly higher $A_{\mathrm{v}}$ distribution but is affected by low number statistics.

the ONC. The three distinct PMS populations found in our CMD and their spatial distribution are not compatible with the presence of an old foreground population of PMS stars. Fang et al. (2017) performed and extensive spectroscopic analysis of 691 "foreground" stars in the Orion A region and confirm that NGC 1980 is not a foreground population. Considering that the candidate foreground population studied by Bouy et al. (2014) is located more than $1 \mathrm{deg}$ south with respect to the peaks of the density maps of the three populations shown in Fig. 3 we think that it is unlikely to be related.

\section{Three discrete episodes of star formation}

In the previous sections we show that differential extinction or distance offsets are very unlikely able to explain the discovered features in the CMD. The presence of unresolved binary and tertiary populations reproduce the CMD morphology only if the underlying mass ratio distribution is rather unusual (Fig. 4).

We here explore the possibility that age is the origin of the discreetness of the color distribution of the PMS in the ONC. We verified that the distance in magnitude between a $1 \mathrm{Myr}$ and a 3 Myr PMS isochrone from Bressan et al. (2012) in the $r, r-i$ CMD is indeed $\sim 0.75$, that is, equal to the the shift in luminosity due to unresolved binaries. This unfortunate fact is at the root of the difficulty to distinguish between the binary hypothesis and the multiple-populations scenarios ${ }^{5}$.

In order to assign ages to the stars belonging to the three distinct PMS populations, we use the measurements presented by Da Rio et al. (2016, D16 hereafter). D16 performed a spectroscopic study of the young stellar population of the Orion A

\footnotetext{
5 We verified that the use of different combinations of filters would not help in this respect.
}

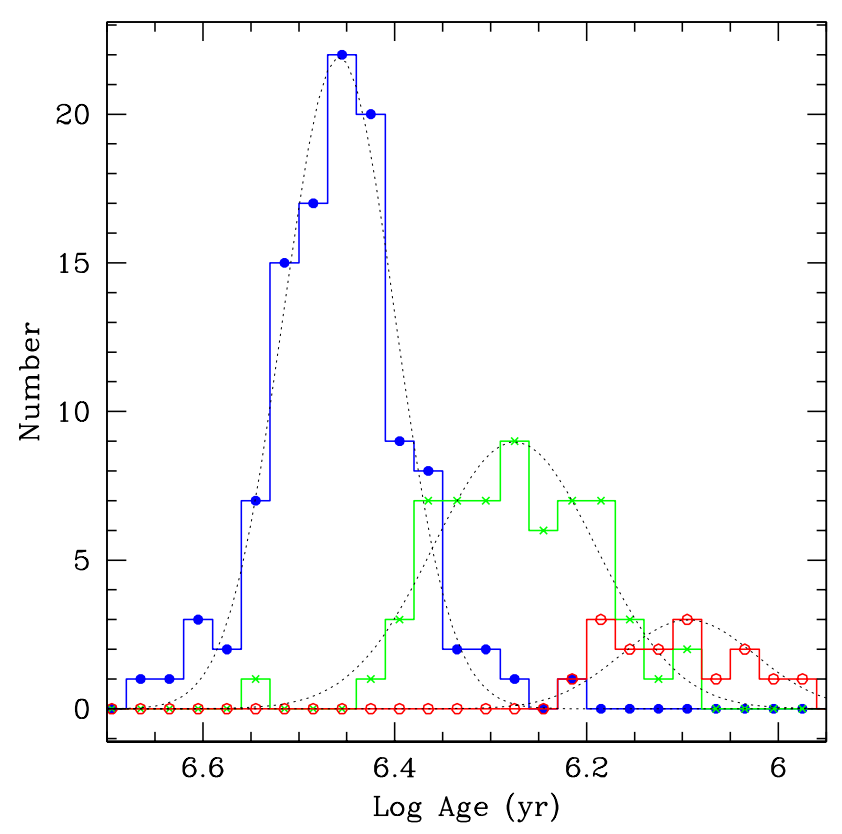

Fig. 6. Distribution of the logarithm of the spectroscopically determined ages for stars from the three populations, old (in blue), young (in green), and very young (in red) with the best Gaussian fits indicated.

molecular cloud with the APOGEE spectrograph. In their work they measured accurate stellar parameters $\left(T_{\mathrm{eff}}, \log g, v \sin i\right)$ and extinctions and concluded that star formation in the ONC proceeds over an extended period of $\sim 3 \mathrm{Myr}$ age. We have crosscorrelated our stars with the catalog of stellar parameters (including age, temperature, and extinction) published in Table 4 of D16. We used these stellar parameters to study the properties of the three candidate populations of PMS stars as selected in Fig. 2. In order to have a sample which is as free as possible from any contamination we removed stars from their study that did not clearly obey a PMS mass-luminosity relation and that, based on the effective temperature, mass, and luminosity, cannot populate the PMS region in the HRD. We were left with 111, 63, and 24 stars for the three sequences (blue, green, and red), respectively.

In Fig. 6 we show the distributions of the ages derived by D16 of the stars in the three samples. These age histograms indicate that the three sequences that we discovered in the photometric study have distinct ages, with the bluer population being the oldest. We have fitted the distributions with Gaussians, using a $\chi^{2}$-minimization technique, to derive the mean and standard deviation $(\sigma)$. As these are distributions of the logarithm of the ages, we then estimated the corresponding distributions of the ages and computed the respective 1- $\sigma$ and 5-95\% intervals. Our results are shown in Table 1.

This finding is consistent with the hypothesis that the formation of the population of PMS stars in the ONC that was thought to be the outcome of a single episode extended over $3 \mathrm{Myr}$ is instead best described by three discrete and sequential episodes of star formation over the same time span.

D16 also provide stellar rotational velocities. However, spectroscopy only provides $v \sin i$, where $i$ is the unknown inclination of the rotation axis on the plane of the sky. As we may assume that $i$ is isotropically distributed in the sky, it is possible to deconvolve, using a Richardson-Lucy method (see Boffin et al. 1993), the $v \sin i$ distributions to obtain the distributions of $v$. These are shown in Fig. 7 and Table 1, where it is clear that not only do the 


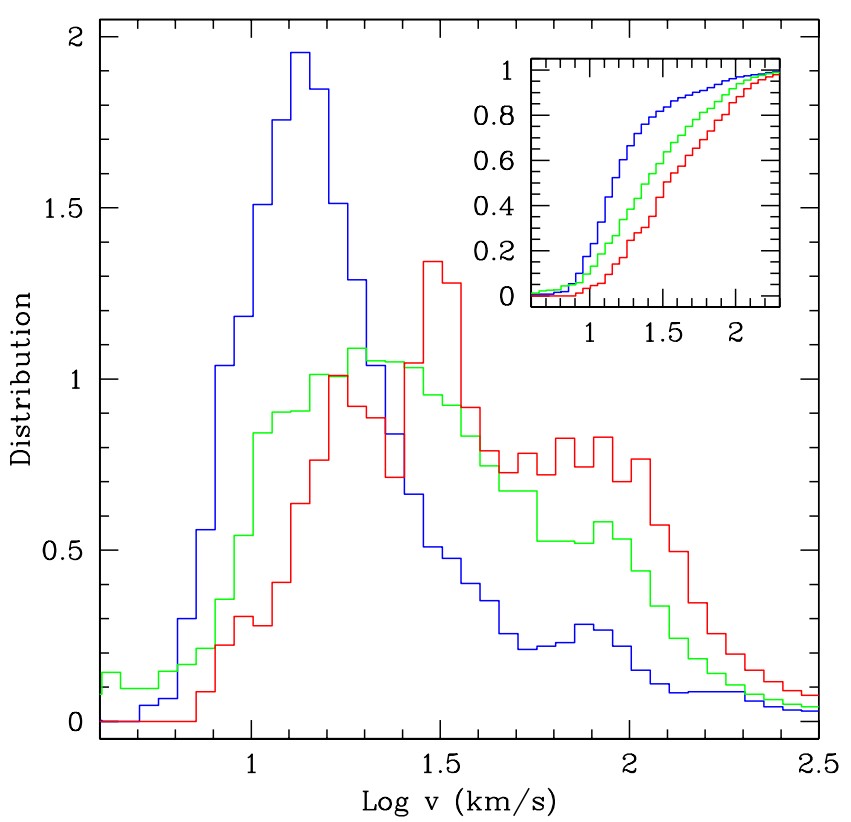

Fig. 7. Distribution of the logarithm of the rotational velocity of the stars from the three populations, old (in blue), young (in green), and very young (in red). The inset shows the normalized cumulative fraction. The Kolmogorov-Smirnov test indicates that the distribution of rotational velocity of the old and younger populations is not extracted from the same parental distribution with more than $5 \sigma$ significance. The same applies when the non-deconvolved rotation velocities are used.

Table 1. Properties of the three populations.

\begin{tabular}{lccc}
\hline \hline & Old & Young & Very young \\
\hline Mean log age (yr) & $6.46 \pm 0.06$ & $6.27 \pm 0.09$ & $6.09 \pm 0.07$ \\
Mean age (Myr) & 2.87 & 1.88 & 1.24 \\
$1-\sigma$ age interval (Myr) & $2.51-3.28$ & $1.55-2.29$ & $1.08-1.53$ \\
5-95\% interval $(\mathrm{Myr})$ & $2.30-3.58$ & $1.37-2.60$ & $1.04-1.63$ \\
Rotational velocity $\left(\mathrm{km} \mathrm{s}^{-1}\right)$ & $14_{-4}^{+6}$ & $25_{-12}^{+25}$ & $35_{-16}^{+36}$ \\
\hline
\end{tabular}

three populations have different ages, but they also have different rotational velocities; the younger the population, the faster its members rotate.

Obviously it could be argued that the age and $v \sin i$ estimations provided in D16 are incorrect if the redder sequences are entirely populated by unresolved multiple systems belonging to the main population. We investigated what would be required to obtain a broadening of the spectral line profile corresponding to $v=25 \mathrm{~km} \mathrm{~s}^{-1}$ (the peak of the young/green population) starting from a binary system containing two stars with $v=14 \mathrm{~km} \mathrm{~s}^{-1}$ (the peak of the old/blue population). It appears that this can only be explained if the binaries have orbital periods between $\sim 2$ and $\sim 12$ months, that is, a very narrow range of periods. Indeed, if the orbital period is greater than $\sim 1$ year then the maximum difference in velocities between the two stars is too small and cannot widen the line and make it appear as a fast rotating star. If, on the other hand, the orbital period is less than 2 months, the difference in velocity is such that a double peaked line profile is detected and, therefore, should have been easily visible in the APOGEE spectra used by D16.

The proponents of the binary hypothesis could also argue that the difference in rotational velocities could be due to tidal effects, as they would spin-up the stars. However, this can only work if the stars were very close to each other, that is, with an orbital period of a few days or less (e.g., Mathieu 1992). Thus, whatever explanation one chooses, one requires a very specific orbital period distribution, unlike what is observed for such stars. Coupling this fact with the narrow distribution of $f(q)$ makes the overall multiplicity scenario very unlikely.

On the other hand, in their seminal work, Rebull et al. (2004) studied the evolution of periods and projected rotational velocities, $v \sin i$, of young PMS stars in the range K5-M2 in several star forming regions, including the ONC. They find a decrease in mean $v \sin i$ as a function of age, and conclude that a significant fraction of all PMS stars must evolve at nearly constant angular velocity during the first 3-5 Myr. Several studies have been performed to look for a suitable explanation of this behavior, which seems to be related to a process of disk locking that might regulate the PMS star angular momentum during early evolution. In short, stars with accretion discs are found to rotate at much slower rates, suggesting that significant angularmomentum-removal mechanisms must operate during the first few Myr of formation (e.g., Davies et al. 2014; Venuti et al. 2017). This scenario, however, has not yet provoked a general consensus (e.g., Herbst \& Mundt 2005; Cieza \& Baliber 2006).

In the context of this paper, we notice that our result on stellar rotation is not unexpected and would support what has already been shown by Rebull et al. (2004). We will investigate the interesting scenario of a relation between stellar rotation and ongoing accretion from a circumstellar disk in a dedicated paper (Beccari et al., in prep.).

\section{Conclusion}

We present a wide-field optical survey of the stellar population in a region of $12^{\circ} \times 8^{\circ}$ in Orion. Our CMD shows the presence of at least two parallel sequences among the PMS stars. The distribution of the $r-i$ colors in the magnitude range $15.5<r<16.5$ reveals the presence of up to three populations. We investigate the origin of these sequences, the existence of which has never before been reported.

We use detailed information (including age, $A_{\mathrm{v}}$, rotational velocities) published in the spectroscopic work of D16 and look for comparative properties of the three populations. We can safely exclude the possibility that differential extinction or different projected distances are responsible for the feature revealed by our CMD. We are hence left with two competitive and equally intriguing explanations: A population of unresolved binaries, or three populations with different ages.

Using a set of Monte Carlo simulations, we tested a range of total binary fractions and mass ratio distributions $f(q)$ needed to reproduce the observed CMD. We find that a flat mass ratio distribution is not able to reproduce the observed color distribution shown in Fig. 2. The observations can be modeled assuming a total of $35 \%$ to $65 \%$ binaries and a $f(q)$ strongly skewed towards high a mass ratio where the majority of the binaries in the ONC have mass ratios $q>0.6$.

This result, if confirmed, would provide the first and most solid constraint on the nature of compact binaries in a population of PMS objects. A dedicated spectroscopic monitoring campaign is urgently required to constrain the multiplicity fraction among these populations. This will certainly allow us to unequivocally distinguish which of the two scenarios best describes reality. Such a population of binaries populating the CMD of the ONC would inevitably challenge many of the previously published studies of stellar populations in the ONC. 
The comparison with the spectroscopic measurements published by D16 provides convincing observational evidence in support of the hypothesis that the ONC contains three populations of PMS stars, with different ages and rotational velocities. In particular we find that the younger the population, the larger the mean rotation velocity.

This result seems not to be unexpected. Rebull et al. (2004) already reported a decrease of $v \sin i$ in the first 5 Myr among PMS stars in the same spectral range studied in this paper and in several star forming regions, including Orion. It has been speculated that the evolution of the angular momentum in solar-type PMS objects might be regulated by actively accreting circumstellar disks through a mechanism of disk-locking. This process would impact the rotational properties of young stars and influence their rotational evolution (Davies et al. 2014). In support of this hypothesis Rebull et al. (2006), using Spitzer mid-IR data for about 900 stars in Orion in the mass range $0.1-3 M_{\odot}$ find that slowly rotating stars are indeed more likely to posses disks than rapidly rotating stars. There is no agreement in the literature on this matter and our dataset will be used in a dedicated paper to further investigate a connection between stellar rotation and ongoing accretion.

While the unresolved binary hypothesis cannot be ruled out, the evidence described so far seems to point toward the first detection of distinct generations of PMS stars in the ONC. Interestingly, using Hubble Space Telescope (HST) observations of the ONC, Reggiani et al. (2011) found that the youngest stars in the cluster are more clustered toward the center, while the oldest ones are distributed almost homogeneously in space. Their figure 12 strongly resembles the populations' distribution shown in our Fig. 3. Along the same line Getman et al. (2014) analyzed the age distribution of the stars in the ONC. Their analysis is based on a new estimator of PMS ages derived from X-ray and nearinfrared photometry. They find that PMS objects in the core of the ONC appear younger and hence formed later than PMS stars in the external regions.

Such a scenario has further interesting implications. In the context of investigating the origin of "blue hook" stars in the globular cluster $\omega$ Cen, Tailo et al. (2015) predicted that these evolved stars originate from the evolution of a rapidly rotating second-generation PMS population whose accretion discs have suffered an early disruption in the dense environment of the cluster's central regions. The result shown in this work may represent the first observational evidence that such a mechanism takes place in the early stages of a cluster's formation.

Since the centers of the spatial distributions of the three populations are not significantly different, we speculate that star formation has been progressing along the line of sight and that the youngest populations formed, on average, further away from us. This view seems consistent with the fact that the $A_{\mathrm{v}}$ distributions for the stars in the different populations are not significantly different, and the youngest population showing a lack of very low$A_{\mathrm{v}}$ sources. The clear decrease in the number of stars belonging to the younger populations, with respect to oldest one, indicates that the overall cluster-formation process is coming to an end, and that the major of activity took place in the beginning when the cluster started to form. This is opposed to the model of accelerated star formation proposed by Palla \& Stahler (2000). In fact, the $1 \sigma$ age intervals from Table 1 indicate that the typical age spreads of the three populations are $\sim 0.5-0.8 \mathrm{Myr}$, which is in excellent agreement with the characteristic dynamical timescale of $7 \times 10^{5} \mathrm{yr}$ for the ONC derived by Tan et al. (2006). Clearly, this prompts a revised look at the formation time-scales of stars in clusters.
Acknowledgements. Based on data collected through ESO programme 096.C0730(A). This research was made possible through the use of the AAVSO Photometric All-Sky Survey (APASS), funded by the Robert Martin Ayers Sciences Fund. C.F.M. acknowledges the ESA Research Fellowship. D.F. acknowledges support from the Italian Ministry of Education, Universities and Research project SIR (RBSI14ZRHR). E.M. was supported by the Spanish Ministry of Economy and Competitiveness (MINECO) under the grant AYA2015-69350-C3-1. AllWISE makes use of data from WISE, which is a joint project of the University of California, Los Angeles, and the Jet Propulsion Laboratory/California Institute of Technology, and NEOWISE, which is a project of the Jet Propulsion Laboratory/California Institute of Technology. WISE and NEOWISE are funded by the National Aeronautics and Space Administration.

\section{References}

Allison, R. J., Goodwin, S. P., Parker, R. J., et al. 2009, MNRAS, 395, 1449 Alves, J., \& Bouy, H. 2012, A\&A, 547, A97

Balog, Z., Siegler, N., Rieke, G. H., et al. 2016, ApJ, 832, 87

Bate, M. R. 2009, MNRAS, 392, 590

Bell, C. P. M., Naylor, T., Mayne, N. J., Jeffries, R. D., \& Littlefair, S. P. 2013, MNRAS, 434, 806

Boffin, H. M. J., Cerf, N., \& Paulus, G. 1993, A\&A, 271, 125

Bouy, H., Alves, J., Bertin, E., Sarro, L. M., \& Barrado, D. 2014, A\&A, 564, A2

Bressan, A., Marigo, P., Girardi, L., et al. 2012, MNRAS, 427, 127

Cardelli, J. A., Clayton, G. C., \& Mathis, J. S. 1989, ApJ, 345, 245

Cargile, P. A., \& James, D. J. 2010, AJ, 140, 677

Cartwright, A., \& Whitworth, A. P. 2004, MNRAS, 348, 589

Cignoni, M., Tosi, M., Sabbi, E., et al. 2010, ApJ, 712, L63

Cieza, L., \& Baliber, N. 2006, ApJ, 649, 862

Correia, S., Duchêne, G., Reipurth, B., et al. 2013, A\&A, 557, A63

Daemgen, S., Correia, S., \& Petr-Gotzens, M. G. 2012, A\&A, 540, A46

Da Rio, N., Robberto, M., Soderblom, D. R., et al. 2009, ApJS, 183, 261

Da Rio, N., Robberto, M., Soderblom, D. R., et al. 2010, ApJ, 722, 1092

Da Rio, N., Tan, J. C., Covey, K. R., et al. 2016, ApJ, 818, 59

Davies, C. L., Gregory, S. G., \& Greaves, J. S. 2014, MNRAS, 444, 1157

De Marchi, G., Panagia, N., Sabbi, E., et al. 2016, MNRAS, 455, 4373

Drew, J. E., Gonzalez-Solares, E., Greimel, R., et al. 2014, MNRAS, 440, 2036

Duchêne, G., \& Kraus, A. 2013, ARA\&A, 51, 269

Fang, M., Kim, J. S., Pascucci, I., et al. 2017, AJ, 153, 188

Getman, K. V., Feigelson, E. D., \& Kuhn, M. A. 2014, ApJ, 787, 109

Gladwin, P. P., Kitsionas, S., Boffin, H. M. J., \& Whitworth, A. P. 1999, MNRAS 302,305

Herbst, W., \& Mundt, R. 2005, ApJ, 633, 967

Hillenbrand, L. A., Hoffer, A. S., \& Herczeg, G. J. 2013, AJ, 146, 85

Irwin, M. J. 1985, MNRAS, 214, 575

Jeffries, R. D., Littlefair, S. P., Naylor, T., \& Mayne, N. J. 2011, MNRAS, 418 , 1948

Köhler, R., Petr-Gotzens, M. G., McCaughrean, M. J., et al. 2006, A\&A, 458, 461

Kounkel, M., Hartmann, L., Tobin, J. J., et al. 2016, ApJ, 821, 8

Luhman, K. L., McLeod, K. K., Goldenson, N. 2005, ApJ, 623, 1141

Mathieu, R. D. 1992, Binaries as Tracers of Star Formation, 155

Menten, K. M., Reid, M. J., Forbrich, J., \& Brunthaler, A. 2007, A\&A, 474, 515

Milone, A. P., Stetson, P. B., Piotto, G., et al. 2009, A\&A, 503, 755

Palla, F., \& Stahler, S. W. 2000, ApJ, 540, 255

Palla, F., Randich, S., Flaccomio, E., \& Pallavicini, R. 2005, ApJ, 626, 49

Palla, F., Randich, S., Pavlenko, Ya. V., Flaccomio, E., \& Pallavicini, R. 2007, ApJ, 659, 41

Parker, R. J., \& Reggiani, M. M. 2013, MNRAS, 432, 2378

Petr, M. G., Coudé du Foresto, V., Beckwith, S. V. W., Richichi, A., \& McCaughrean, M. J. 1998, ApJ, 500, 825

Rebull, L. M., Wolff, S. C., Strom, S. E. 2004, AJ, 127, 1029

Rebull, L. M., Stauffer, J. R., Megeath, S. T., Hora, J. L., \& Hartmann, L. 2006, ApJ, 646, 297

Reggiani, M., Robberto, M., Da Rio, N., et al. 2011, A\&A, 534, A83

Reipurth, B., Guimarães, M. M., Connelley, M. S., \& Bally, J. 2007, AJ, 134 2272

Reipurth, B., Clarke, C. J., Boss, A. P., et al. 2014, Protostars and Planets (University of Arizona Press), 267

Schlafly, E. F., Green, G., Finkbeiner, D. P., et al. 2014, ApJ, 786, 29

Spiegler, G., \& Juris, K. 1931, Phot. Korr., 67, 4

Tailo, M., D'Antona, F., Vesperini, E., et al. 2015, Nature, 523, 318

Tobin, J. J., Hartmann, L., Furesz, G., Mateo, M., \& Megeath, S. T. 2013, ApJ, 773,81

Tan, J. C., Krumholz, M. R., \& McKee, C. F. 2006, ApJ, 641, L121

Venuti, L., Bouvier, J., Cody, A. M., et al. 2017, A\&A, 599, A23

Ward-Duong, K., Patience, J., De Rosa, R. J., et al. 2015, MNRAS, 449, 2618 